\section{NECESIDAD DE EVALUAR LAS GUÍAS CLÍNICAS PERUANAS DE TRATAMIENTO PARA TRASTORNOS MENTALES}

\section{THE NEED FOR EVALUATING THE PERUVIAN CLINICAL GUIDELINES FOR TREATMENT OF MENTAL DISORDERS}

\author{
Juan Antonio Gálvez-Buccollini'1,2,3,a \\ Fabián Fiestas $3,4, \mathrm{~b}$
}

Sr. Editor. La Organización Panamericana de la Salud (OPS) estima que el $90 \%$ de los problemas neuropsiquiátricos, pueden ser atendidos en el nivel primario de atención (1). Esta estimación es relevante para varios países como el Perú, donde este tipo de problemas constituyen la primera causa de pérdidas por discapacidad y muerte prematura, y el número de psiquiatras es reducido y mal distribuido a través de sus regiones. Así, la participación de médicos no psiquiatras en la detección y tratamiento de estos problemas, representa una solución a este desventajoso escenario. Sin embargo, es frecuente que los médicos no psiquiatras tengan dificultades en reconocer los síntomas, establecer un diagnóstico e iniciar un tratamiento adecuado para los problemas de salud mental (2). Esta situación lleva a que muchos de los pacientes que logran acceder a los servicios de salud -lo que es ya un enorme desafío- consigan ayuda médica muy limitada para estos problemas.

Uno de los pasos iniciales que se deben dar si se quiere implementar y desarrollar un sistema comunitario de salud mental, es contar con guías clínicas de tratamiento (GCT). Esto es porque las GCT -si son construidas a través de un estricto proceso científico y pasan por un proceso de concertación con actores locales de amplia experiencia en el área- entregan información concreta, contextualizada y basada en la mejor evidencia científica disponible. Con las GCT, los médicos no especialistas podrían identificar y tratar satisfactoriamente trastornos mentales, así como derivar a cuidado especializado aquellos casos que así lo requieran ${ }^{(3)}$.

\footnotetext{
Harvard South Shore Psychiatry Residency Training Program, Harvard Medical School. Boston, EEUU.

2 Veterans Affairs Boston Healthcare System. Boston, EEUU.

3 Red para la Acción y Avance de la Salud Mental y Psiquiatría (Red AVANSE-PSI). Lima, Perú.

4 Unidad de Análisis y Generación de Evidencias en Salud Pública Instituto Nacional de Salud. Lima, Perú.

a Médico Cirujano; ${ }^{b}$ Médico Epidemiólogo

Recibido: 04-11-11 Aprobado: 09-11-11
}

El Ministerio de Salud del Perú (MINSA) reconoce la importancia de las GCT. Por lo cual, publicó entre el 2006 y el 2008, GCT para depresión, psicosis, violencia basada en género y para problemas relacionados con el uso de sustancias psicoactivas (http://www.minsa.gob. pe/portada/est_san/saludmental.htm). Sin embargo, estas guías necesitan una revisión crítica con relación a sus recomendaciones. Mencionaremos aquí solo algunas observaciones a la GCT de depresión (4), por ser uno de los problemas de salud mental más frecuentes y de tratamiento más accesible en el nivel primario de atención.

Por ejemplo, en esta guía se recomiendan dos esquemas principales de tratamiento que pueden ser usados en el nivel de atención primaria. El primer esquema incluye fluoxetina, $20 \mathrm{mg}$, con la posibilidad de aumentar a $40 \mathrm{mg}$ diarios si no hay repuesta con la dosis inicial. El segundo esquema incluye una prueba terapéutica de sertralina, $50 \mathrm{mg}{ }^{(4)}$. Si el paciente no responde al esquema iniciado, el siguiente paso es referirlo a un tratamiento especializado. Sin embargo, un ensayo terapéutico completo significa usar la medicación a dosis máxima (o máxima tolerable) por un tiempo establecido (5). Entonces, si seguimos el esquema propuesto por la actual GCT, se estaría refiriendo al paciente de forma precoz al especialista sin establecer con claridad si realmente el paciente no respondió al tratamiento o si la dosis fue insuficiente, particularmente si hubo una respuesta parcial. Debido a que en muchas partes del Perú los servicios especializados en salud mental no existen o son muy limitados, una referencia precoz al especialista no sería lo más idóneo desde la perspectiva de la salud pública.

De otro lado, la discusión que la GCT hace sobre efectos adversos e interacciones de los antidepresivos es limitada. Por ejemplo, no se menciona los potenciales efectos adversos en la función sexual a pesar de ser un problema prevalente, especialmente con los ISRS (26-80\%), lo que puede afectar significativamente la adherencia al tratamiento y la calidad de vida. Asimismo, la GCT menciona que los ISRS no disminuyen el umbral convulsivo a pesar de que la evidencia muestra que estos medicamentos sí lo hacen, aunque en menor medida que los antidepresivos tricíclicos (ATC) ${ }^{(6)}$.

Más adelante, la actual GCT considera el uso de la combinación de ATC y los inhibidores de la monoaminoxidasa (IMAO) en los casos de depresión refractaria al tratamiento. Si bien existen informes del uso de IMAO en combinación con algunos ATC, no existen ensayos clínicos doble ciego randomizados que confirmen seguridad y eficacia. Por lo cual, la recomendación estándar es no combinar ATC con 
IMAO ${ }^{(6)}$. En concordancia, la guía de depresión de la OMS tampoco incluye esta recomendación. De hecho, existe evidencia que la combinación ATC-IMAO puede resultar en graves complicaciones (Ej. el síndrome serotoninérgico) que pueden llevar a la muerte. Por lo cual, se debe considerar revisar la idoneidad de incluir esta recomendación en una GCT.

En la sección de complicaciones de la GCT se menciona que el $15 \%$ de los pacientes con depresión termina suicidándose. Sin embargo, no se discute cómo realizar una adecuada evaluación del riesgo suicida siendo esta parte esencial en cualquier evaluación de salud mental. Además, si bien se señala inicialmente que el diagnóstico de depresión es clínico, en la sección de ayuda diagnóstica se mencionan como exámenes útiles al SPECT y la prueba de supresión de dexametasona. Estos procedimientos pueden ser informativos y útiles en el área de investigación, pero no tienen ninguna relevancia o aplicación estandarizada en la práctica clínica. De esta manera, se podría eventualmente inducir a un malgasto de los escasos recursos con que los pacientes y el sistema de salud cuentan en nuestro país.

Todo lo expuesto nos obliga a recomendar una revisión y actualización urgente de las GCT peruanas para trastornos mentales. Estas GCT deben ser construidas usando los procesos científicos más rigurosos y ser adaptadas o contextualizadas a las circunstancias específicas asistenciales y de disponibilidad de recursos en salud que tiene nuestro país. EI MINSA, en coordinación con las instituciones especializadas en metodologías de revisiones sistemáticas y guías clínicas (como el Instituto Nacional de Salud), e instituciones especializadas en salud mental, (como el Instituto
Nacional de Salud Mental, DEVIDA, entre otras) deben iniciar estas acciones, las cuales, de llevarse a cabo, lograrán hacer más accesible un cuidado de calidad y estandarizado en salud mental a toda la población.

\section{Conflictos de interés}

Los autores declaran no tener conflicto de intereses.

\section{REFERENCIAS BIBLIOGRÁFICAS}

1. Organización Panamemericana de la Salud. Marco de Referencia para la implementación de la Estrategia Regional de Salud Mental. Washington DC, EE.UU.: OPS; 2011.

2. Wittchen HU, Höfler M, Meister W. Prevalence and recognition of depressive syndromes in German primary care settings: poorly recognized and treated? Int Clin Psychopharmacol. 2001;16(3):121-35.

3. Zeiss AM, Karlin BE. Integrating mental health and primary care services in the Department of Veterans Affairs health care system. J Clin Psychol Med Settings. 2008;15(1):73-8.

4. Ministerio de Salud del Perú. Guía de Práctica Clínica en Depresión. Lima: Ministerio de Salud; 2008.

5. Sadock BJ, Sadock VA. Kaplan \& Sadock's Synopsis of Psychiatry: Behavioral Science/Clinical Psychiatry. $9^{\text {th }}$ ed. Philadelphia: Lippincott Williams and Wilkins; 2003.

6. Haddad PM, Dursun S. Neurological complications of psychiatric drugs: Clinical features and management. Hum Psychopharmacol Clin Exp. 2008;23(Suppl1):15-26.

Correspondencia: Juan Antonio Gálvez-Buccollini Abanto Clinical Fellow in Psychiatry, Harvard Medical School. Boston MA, EE.UU.

Teléfono: (508) 857-7906

Correo electrónico: Juan_Galvez-Buccollini@hms.harvard.edu 